\title{
Subjective well-being in China: how much does commuting matter?
}

\author{
Zhenjun Zhu' ${ }^{1}$ Zhigang $\mathrm{Li}^{2} \cdot$ Hongsheng $\mathrm{Chen}^{3}$ (D) $\cdot$ Ye $\mathrm{Liu}^{3} \cdot$ \\ Jun Zeng'
}

(C) The Author(s) 2017. This article is an open access publication

\begin{abstract}
With rapidly increasing urbanization and motorization in China, the effect of commuting on residents' subjective well-being (SWB) is likely growing. We used 13,261 individual, 124 city, and 401 neighbourhood samples from the 2014 China Labour-Force Dynamics Survey (CLDS 2014) and applied multilevel mixed-effects ordered probit regressions to investigate the relationship between commuting and SWB. We found huge differences between urban and rural areas in relation to commuting. Urban respondents' daily average commuting time was $0.56 \mathrm{~h}$ while rural respondents' daily average commuting time was $0.41 \mathrm{~h}$. Further, the daily average commute time for residents living in cities with high urbanization rates $(>70 \%)$ was longer than for those living in cities with low urbanization rates $(<70 \%)$. The subjective well-being of residents who commute by walking or cycling was significantly lower than that of those who commute by other transportation modes. The regression results indicated that the longer the commute time, the lower the subjective well-being. Among residents who live in rural areas or cities with low urbanization, subjective well-being was more easily affected by commuting time.
\end{abstract}

Hongsheng Chen

hongsheng.chen2006@163.com

Zhenjun Zhu

zhuzhenjun@seu.edu.cn

Zhigang Li

zhigangli@whu.edu.cn

Ye Liu

liuye25@mail.sysu.edu.cn

Jun Zeng

zengjun@seu.edu.cn

School of Transportation, Southeast University, Si Pai Lou No. 2, Nanjing 210096, China

2 School of Urban Design, Wuhan University, Ba Yi Road No. 299, Wuhan 430072, China

3 School of Geography and Planning, Sun Yat-Sen University, Xingang Xi Road,

Guangzhou 510275, China 
Commuting time was also found to influence residents' job satisfaction and family life satisfaction, which in turn influence SWB. China's current development mode ignores the traffic needs of vulnerable groups. Therefore, future traffic construction should increase its prioritization of these vulnerable transportation groups.

Keywords Commuting time - Commuting mode - Subjective well-being - Urbanization · Migrants · China

\section{Introduction}

Commuting is an important aspect of urban lives. An increasing amount of research has focused on the relationship between commuting and urban residents' subjective well-being (SWB) (Kroesen 2014; Dickerson et al. 2014), or how people experience their quality of life, including life satisfaction and emotional responses (Diener 1984; Liu et al. 2017). Every weekday, hundreds of millions of employed Chinese travel to work, especially in China's big cities (Zhao et al. 2011a, b; Sun et al. 2016). In recent years, with rapidly increasing urbanization and motorization in China, traffic congestion has worsened. With population growth in the cities and the expansion of the urban spatial structure, workplaces and residences are becoming increasingly distant, and commuting times are increasing accordingly (Yang and Gakenheimer 2007; Lin et al. 2015; Sun et al. 2016). It has been calculated that costs related to traffic congestion in Beijing reduce the municipal gross domestic product by 4-7\% (Creutzig and He 2009). Increased commute times not only increase costs for commuters but also affect their SWB (Ettema et al. 2011; Ferenchak and Katirai 2015). For example, Ye and Titheridge (2015), using specially designed web-based and paper-based surveys, gathered data from Xi' an showing that commuting characteristics (e.g. commuting mode choice), significantly influence commuting satisfaction, which in turn significantly affects SWB. In addition, although leisure travel has a significant effect on residents' SWB (Mccabe and Johnson 2013; Mccabe et al. 2010), leisure activities mainly occur during the holidays, and compared with leisure activities, most city residents need to commute every day. Thus, workday commutes have a constant impact on residents' SWB. Investigating the relationship between commuting and SWB in China will help to deepen our understanding of the impact of commuting on the lives of Chinese residents.

Although many studies have found a significant correlation between commuting and SWB (Abou-Zeid et al. 2012; Olsson et al. 2013; Ferenchak and Katirai 2015), there are still some research gaps. First, most previous studies on this relationship have been conducted in developed countries. As we know, there is a big difference in urban environments between developed and developing countries; in particular, many developing countries are still in a stage of rapid urbanization with the concurrent rapid development of urban traffic. Especially in China, the traffic problem is becoming increasingly serious (Wang et al. 2014; Linn et al. 2016). Most urban residents or workers are facing traffic problems and being forced to adapt to ever-increasing congestion. Second, the conclusion reached by previous studies that a significant correlation exists between commuting and SWB has been mostly based on surveys of single cities (Abou-Zeid and Ben-Akiva 2011; Ettema et al. 2010). Few studies have compared the relationship between commuting and SWB in different types of cities (e.g. by urbanization rate). In China, different cities with different urbanization rates have different transport facilities and spatial structures (Chen et al. 2008). There are also large differences in residents' commute times (Wang and Chai 2009; 
Zhao et al. 2011a, b). For instance, Yang and Gakenheimer (2007) noted that China's current urban development mode tends to lengthen trips and lead to increased congestion in the context of growth and motorization. Zhao et al. (2010) found that sprawling expansion with a low degree of self-contained development and low-density land use tends to increase the need for long-distance commuting from the city outskirts to the central urban area of Beijing. In general, residents' daily commute times and distances are longer in cities with higher urbanization rates than those with lower urbanization rates. As a result, residents' acceptance of commute times and distances differs in cities with different urbanization rates. Moreover, the happiness of residents in cities with different urbanization rates has also been shown to differ significantly. For example, Gunatilaka (2011) found that happiness has not increased along with China's recent rapid urbanization and economic growth, and residents living in rural areas report higher SWB than their richer urban counterparts. Further, the SWB of people living in big cities with high urbanization rates may be lower than that of people living in small cities with low urbanization rates. Therefore, to better analyse the impact of commuting on urban residents' SWB, this study divided the sampled cities into three urbanization rates-50, 50-70, and 70\%-which represent low, medium, and high urbanization rates, respectively. Third, it is well known that there are significant differences in the commuter patterns of different groups (e.g. those with different incomes and those living in different neighbourhoods). However, there no previous study has compared the relationship between commuting and SWB between such groups (e.g. between locals and migrants).

To fill these knowledge gaps, this study used Chinese national survey data to investigate the relationship between commuting and residents' SWB in cities with different urbanization rates and among groups with different hukou (locals with local hukou and migrants without local hukou). Hukou is one of the main factors affecting China's social inequalities (Chan and Zhang 1999). Zhao and Howden-Chapman (2010) found that the hukou system can reduce migrant families' chances of finding employment and increase their time spent commuting. Residents with different hukou will have different job-housing relationships (Na et al. 2017; Li and Liu 2016). The rest of this paper is organized as follows. "Relationship between commuting and subjective well-being" section provides a brief overview of the literature on the relationship between commuting and SWB (worldwide). In "Methods" section, we introduce the data and statistical models used in this study; then, "Results" section presents the results. "Discussion and Conclusion" sections discuss these results and give a conclusion, respectively.

\section{Relationship between commuting and subjective well-being}

Subjective well-being (SWB) refers to how people experience the quality of their own life and includes life satisfaction and emotional reactions (Diener 1984; Veenhoven 2015; Liu et al. 2017). Increasingly, studies have regarded SWB as a valid proxy for personal objective welfare (Frey and Stutzer 2002a, b; Layard 2010; Kahneman and Krueger 2006; Tella and MacCulloch 2006; Clark et al. 2008a, b; Stutzer and Frey 2010; MacKerron 2012; Crespo and Mesurado 2015). Many studies have investigated the impact of socioeconomic and built-environment factors on SWB. Regarding personal-level factors, it has been shown that low income, limited education, poor health, separation, unemployment, and lack of social contact are strongly negatively associated with SWB (Dolan et al. 2008; Kahneman and Deaton 2010). For regional- and national-level factors, many studies 
have examined the 'Happiness-Income Paradox' or the 'Easterlin paradox' (Easterlin and Angelescu 2009; Stevenson and Wolfers 2008). Easterlin and Angelescu's (2009) 'Happiness-Income Paradox' suggests that no significant relationship exists between improved happiness and GDP growth per capita. Further, many scholars have argued that a person's SWB depends to a large degree on relative income (Mcbride 2001). Neighbourhoods and built environments also have direct and indirect effects on SWB. For example, Tella and Macculloch (2005) found that, between 1975 and 1997, environmental degradation and crime were negatively correlated with people's happiness in OECD countries. Ludwig et al. (2012) found that moving from high-poverty to low-poverty neighbourhoods improved residents' SWB. Many studies have found that local air pollution and noisy living environments significantly diminish residents' SWB (Welsch 2006; MacKerron and Mourato 2009). Other findings have highlighted the effects of physical neighbourhood environments (e.g. housing price and quality) as well as neighbourhoods' social environments (e.g. sense of community) on SWB (Farrell et al. 2004).

However, in the existing literature, the relationships of commuting time and commuting mode with SWB are controversial (Choi et al. 2013; Dickerson et al. 2014; Morrow 2010; Morris 2011; Munford 2014; Kroesen 2014; Sweet and Kanaroglou 2016; Kroesen, 2014). Although some studies have concluded that commuting time has no effect on SWB, we might nevertheless consider it a common sentiment that commuting on congested roads is unpleasant. There are also research findings to support this. Choi et al. (2013), using Gallup-Healthways survey data for the United States, found a significant negative relation between commute time and SWB. Kahneman et al. (2004) used a day reconstruction method and found that the most disliked and least popular activity among 16 major human activities was commuting. Stutzer and Frey (2008), using panel data from the German Socioeconomic Panel Study, found that people with longer commuting time have lower SWB. Roberts et al. (2011), using annual data from the British Household Panel Survey, found that commuting has a considerable detrimental impact on the well-being of women, which may be due in part to their shorter working hours. Wheatley (2014), again using British Household Panel Survey data, found that longer commutes generated dissatisfaction among men and women, as did shorter commuting among women due to inequality in household division of labour; this is consistent with conclusions obtained by Roberts et al. (2011), and Smith (2013). Part of understanding these findings is appreciating that the relationship between commuting time and well-being varies depending on both location and respondent features. For instance, Morris (2011) put forward that variables at the regional level, such as traffic congestion, transfer frequency, and car cost are not significantly related to SWB, while variables at the individual level, such as income, are.

Besides commuting time, previous studies have found that the commuting mode is another important factor that affects residents' SWB. For example, Gatersleben and Uzzell (2007), using data from a survey done at the University of Surrey, found that car commuting is more stressful than other commuting modes, at least partly because public transport commuting is boring. Duarte et al. (2010), using data from several European cities, again found that commuting mode choice has a significant impact on well-being: subway commuters are happier than private car commuters. Abou-Zeid and Ben-Akiva (2011), using a web-based cross-sectional survey conducted with a convenience sample of commuters, found that commuters travelling by non-motorized modes are happier than those travelling by car or public transport. Smith (2013), using data from a web-based survey of workers in Portland, Oregon, United States, found that walking and cycling commuters have significantly higher commute well-being than transit and car commuters. Zhao and Lee (2013), surveying 2000 North Americans who commute to work or school, 
found that most measures of commuting happiness and satisfaction are heavily dependent on comfort and reliability ratings of commuting modes. Abou-Zeid and Fujii (2016), using the results of an experiment conducted by the Massachusetts Institute of Technology requiring temporary use of public transport to commute to work by habitual car drivers, observing an increase in the average level of satisfaction with the commute among those participants.

In addition, scholars have increasingly used the life-oriented approach to better understand travel behaviour and daily life (Zhang and Acker 2017; Zhang 2017; Beige and Axhausen 2017). For example, Gerber et al. (2017) found that residents had higher life satisfaction after relocating from Luxembourg to neighbouring countries with lower real estate prices, despite increased commute times. Sascha et al., using data from the Dutch GeluksWijzer (Happiness Indicator), found that people typically feel less well when commuting than at home; this negative difference was largest when commuting using public transportation and smallest when commuting by bike. Wang et al. (2017) analysed holiday travel behaviour dynamics using IMTI (Integrated Multimodal Travel Information), based on the life-oriented approach, and found that life biography is affected not only by personal life course but also by the external background of the times. Delbosc and Nakanishi (2017) found that among many millennials in Australia, few showed a strong preference for cars, but as they approached adult milestones (e.g. having children, finding suitable housing near public transit), they came to feel they had no choice but to own a car. Yu et al. (2017) developed the DU-DCLH model to describe mobility over the course of life and confirmed the influence of time-constant and time-varying preferences for residential neighbourhoods. In travel behaviour analysis using life-oriented approaches, travel behaviours not only result from life choices but also affect people's lives.

This study aims to fill the research gap in commuter-happiness relations in China under rapid urbanization. On the one hand, this study compares the commuting-SWB relationship in cities with different levels of urbanization, which will improve our understanding of the real impact of urban transport development on the daily lives of people in China. On the other hand, this study compares the commuting-SWB relationship among different hukou holders, which will help us to better understand differences in the commuting patterns and effects of different groups in China's specific social context.

\section{Methods}

\section{Data}

The data used for this study were obtained from the 2014 China Labour-Force Dynamics Survey (CLDS 2014), conducted by the Centre for Social Science Surveys at Sun Yat-Sen University, in Guangzhou. The CLDS 2014 was a nationwide cross-sectional survey covering 29 mainland provinces and municipalities (excluding Tibet and Hainan), with multistage clustering and a stratified probability sampling strategy. The CLDS 2014 established a tracking database with three levels: individual, neighbourhood, and city. The CLDS 2014 questionnaires covered individual socioeconomic status, commuting situation, working conditions, and other demographic information, all of which were used in the analysis. Additionally, data collection was conducted in a community-based manner, using a random sampling method. The professional staff went to the interviewees' homes with their consent, using face-to-face surveys, to reduce the impact of other potential factors on 
respondents' SWB (e.g. the weather). Due to the hierarchical structure of the CLDS 2014 data, three-level mixed-effects ordered probit regressions were performed to investigate the association between commuting and SWB. In the data analysis, samples with missing data or unemployed respondents were excluded. This left 13,710 individual samples, 124 city samples, and 401 neighbourhood samples for regression analysis.

\section{Models and variables}

In the regression model, the dependent variable was SWB. In the CLDS 2014, respondents were asked to rate their SWB on a five-point Likert-type scale ranging from very unhappy (1) to very happy (5). In the CLDS 2014 survey, respondents gave seven main commuting modes: walk, bicycle, motorcycle, bus, subway, taxi, and private car. In this study, we grouped bus, subway, and taxi as public transport. The independent variables were daily commute time (continuous variable), public transport mode (ref: other transportation modes), and walking/bicycling (ref: other transport modes). Most existing studies have focused on the impact of socioeconomic factors on residents' SWB while failing to consider that commute time might also be an important factor affecting SWB. Especially in China's large cities (e.g. Beijing), with high levels of urbanization, some residents spend as much as 4-6 h a day commuting (see http://bj.jjj.qq.com/a/20160926/016439.htm). Long commute times make people feel greater pressure in urban life, which could negatively influence SWB. Moreover, suitable commute times differ for different transport modes. However, there are few comparative studies on the effect of the commute times of different transport modes on SWB. For example, since private cars are more comfortable than public transport, does commute time have no significant effect on the SWB of those who use private transport? What is a reasonable commute time for public transport? These questions require further investigation. Therefore, we chose those variables as the explanatory variables. The controlled variables include age (continuous variable), gender (ref: female), marital status (ref: single, divorced, or widowed), educational attainment (ref: primary school and below), logarithm of annual personal income (continuous variable), and hukou status (ref: non-local hukou status).

Some existing studies have shown that city/neighbourhood characteristics are closely related to residents' SWB (Liu et al. 2017). Therefore, to control city/neighbourhood factors, this study used a multilevel mixed-effects ordered probit regression to analyse the relationship between commuting and SWB. We divided the 124 cities in China into three types: cities with urbanization rate $<50 \%$, cities with $50 \%<$ urbanization rate $<70 \%$, and cities with urbanization rate $>70 \%$. In this study, urbanization rate is the proportion of the population living in urban areas to the total population. In China, cities with higher urbanization rate tend to have a higher level of economic development (e.g. Beijing, Shanghai, Shenzhen). In addition, we analysed the relationship between commuting and SWB for residents with different hukou ('local people', who own a local hukou, and 'migrants', who do not). The lifestyles and SWB of local residents in a given city are generally higher than those of migrants (Wong et al. 2007); therefore, the impact of commuting on their SWB may be different as well. 


\section{Results}

\section{Descriptive statistics}

According to the CLDS 2014, there are huge differences between urban and rural areas in relation to commuting (see Table 1). Urban respondents' daily average commute time is $0.56 \mathrm{~h}$, while rural respondents' daily average commute time is $0.41 \mathrm{~h}$. The daily average commute time of urban respondents who live in cities with high urbanization rates $(>70 \%)$ is longer than for those living in cities with low urbanization rates $(<70 \%)$. For rural respondents, those living in cities with medium urbanization rates $(50-70 \%)$ have shorter commutes than their counterparts. Walking is the main commuting mode for urban and rural respondents, accounting for 33.00 and $57.46 \%$, respectively. As urbanization rate increases, the proportion of residents commuting by walking gradually decreases. The proportion of commuters using motorcycles is much smaller in cities with high urbanization rates than in those with medium and low urbanization rates. This is mainly because government motorcycles are still very common in China's small and medium-sized cities but are forbidden in large cities because of the increasingly serious traffic problems they cause (Deng et al. 2009). The proportion of commuters using private cars is much larger in urban areas than in rural areas (13.74 vs $3.46 \%$ ). As urbanization rate increases, private car use increases as well. Additionally, commuting mode also varies widely by urbanization rate: the higher the urbanization rate, the greater the proportion of public transport modes (bus, subway, taxi) used for commuting.

Table 2 shows the relationship between transportation modes and respondents' SWB. In urban areas, the SWB of respondents commuting by private car is higher than for those commuting by other transportation modes. In rural areas, the SWB of respondents commuting by subway and walking is lower than for those commuting by other transportation modes. Table 3 shows the relationship between commuting time and respondents' SWB by different transportation modes. In urban areas, for commuters using private cars and taxis, respondents with low levels of SWB commute longer than those with high levels of SWB. In rural areas, for commuters who walk, use bicycles, or ride motorcycles, the commuting time of respondents with low levels of SWB is significantly longer than those with high levels of SWB.

\section{The impact of commuting on residents' SWB}

Table 4 shows the multilevel mixed-effects ordered probit regression results for commuting impact on residents' SWB. Model 1 gives the results for commuting variables and SWB for respondents living in urban areas, and model 2 for respondents living in rural areas. In model 1, traffic variables are not significantly related to the respondents' SWB. In model 2, the results show that the longer one's commute time, the lower one's SWB (coefficient $=-0.108, p<0.01$ ); further, the SWB of respondents commuting by walking and bicycle is more likely to be lower than that of those commuting by other means (coefficient $=-0.090, p<0.01$ ). In addition, for urban respondents, married (coefficient $=0.381, p<0.01)$ and higher-income (coefficient $=0.091, \quad p<0.01$ ) respondents, respectively, have higher SWB than unmarried and lower-income respondents. Compared to respondents with a primary school education or less, respondents with more education (junior high school: coefficient $=0.132, p<0.05$; senior high school: coefficient $=0.188, p<0.01$; college and above: coefficient $=0.376, p<0.01$ ) have 


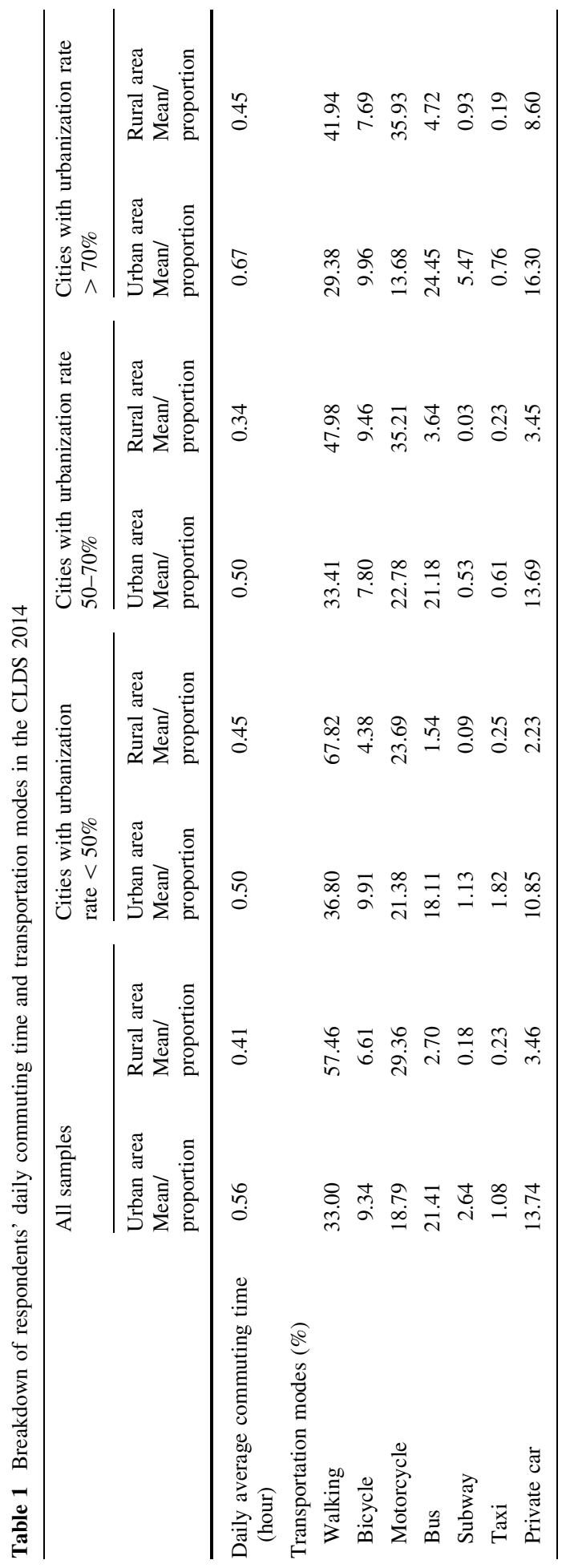


Table 2 The relationship between transportation modes and respondents' subjective wellbeing

\begin{tabular}{lll}
\hline Transportation modes & \multicolumn{2}{l}{ Subjective well-being } \\
\cline { 2 - 3 } & Urban area & Rural area \\
\hline Walking & 3.79 & 3.58 \\
Bicycle & 3.80 & 3.72 \\
Motorcycle & 3.72 & 3.73 \\
Bus & 3.82 & 3.75 \\
Subway & 3.84 & 3.47 \\
Taxi & 3.90 & 3.80 \\
Private car & 4.07 & 3.93 \\
\hline
\end{tabular}

higher SWB. Male urban respondents have lower SWB than female urban respondents (coefficient $=-0.139, p<0.01$ ). For rural respondents, the results for the control variables are almost the same as those in the urban respondent model. Beyond that, for rural respondents, being older and having a local hukou are associated with higher SWB.

Next, we analysed the results after dividing the 124 cities surveyed in CLDS 2014 into three types by urbanization rate, as described earlier. The results in Table 5 show that it is only in less urbanized cities (urbanization rate $<70 \%$ ) that commuting time has a significant and negative effect on residents' SWB - the longer the commute time, the less likely residents are to have a higher level of SWB (urbanization rate $<50 \%$ : coefficient $=-0.118, p<0.01 ; 50 \%<$ urbanization rate $<70 \%$ : coefficient $=-0.079$, $p<0.10$ ). This is probably because in big cities with high levels of urbanization, people are more accustomed to long commutes since there are more traffic jams and longer commuting distances. Thus, SWB does not change rapidly as commuting time increases. However, in small and medium-sized cities with lower levels of urbanization, commuting distance and time are relatively short; thus, if commuting time increases, SWB is more likely to be affected. In cities with medium urbanization rates (50-70\%), the SWB of commuters who walk or ride bicycles is significantly lower than that of commuters who use motor vehicles (coefficient $=-0.093, p<0.05$ ). This could be related to the unfriendly walking and cycling environments of such cities (e.g. the imperfect walking systems and the mix of bike lanes and motorways).

We conducted a regression analysis of the impact of commuting on the SWB of migrants (without local hukou) and local residents (with local hukou), as shown in Table 6. For both migrants and local people, commuting time is significantly and negatively associated with SWB (coefficient $=-0.079, p<0.10$; coefficient $=-0.081, p<0.01$ ). It is only for local people that commuting by walking/cycling makes it less likely to have a higher level of SWB (coefficient $=-0.062, p<0.05$ ). We also performed a multilevel mixed-effects ordered probit regression on traffic's impact on residents' SWB by transport mode (see Tables 7 and 8). For respondents in urban areas, only commuting time has a negative effect on the SWB of those who walk or ride bicycles (coefficient $=-0.118$, $p<0.10$ ). For respondents in rural areas, commuting time has a negative effect on the SWB of those who walk/ride bicycles (coefficient $=-0.086, p<0.05$ ) or ride motorcycles (coefficient $=-0.280, p<0.01$ ). 


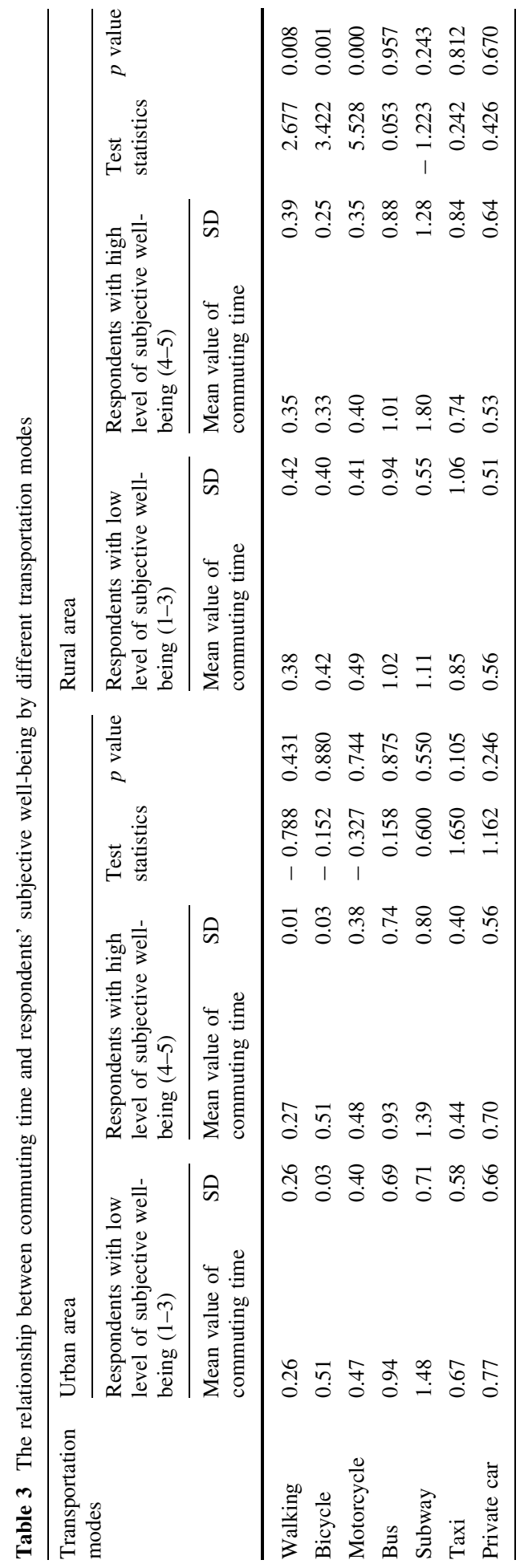


Table 4 Multilevel mixed-effects ordered probit regression results of the impact of commuting on residents' subjective well-being

\begin{tabular}{|c|c|c|c|c|}
\hline & \multicolumn{2}{|c|}{ Model 1: urban area } & \multicolumn{2}{|c|}{ Model 2: rural area } \\
\hline & Coefficient & SE & Coefficient & SE \\
\hline \multicolumn{5}{|l|}{ Traffic variables } \\
\hline Daily commuting time & -0.043 & $(0.033)$ & $-0.108 * * *$ & $(0.030)$ \\
\hline Public transport means (ref: Other transport means) & -0.047 & $(0.048)$ & 0.001 & $(0.075)$ \\
\hline Walk/bicycle (ref: Other transport means) & -0.017 & $(0.042)$ & $-0.090^{* * *}$ & $(0.031)$ \\
\hline \multicolumn{5}{|l|}{ Control variables } \\
\hline Age & -0.001 & $(0.002)$ & $0.003 * *$ & $(0.001)$ \\
\hline Gender (ref: female) & $-0.139 * * *$ & $(0.034)$ & $-0.045^{*}$ & $(0.026)$ \\
\hline Marital status (ref: single, divorced or widowed) & $0.381 * * *$ & $(0.048)$ & $0.176 * * *$ & $(0.042)$ \\
\hline \multicolumn{5}{|l|}{ Educational attainment (ref: primary school and below) } \\
\hline Junior high school & $0.132 * *$ & $(0.061)$ & $0.072 * *$ & $(0.029)$ \\
\hline Senior high school & $0.188 * * *$ & $(0.065)$ & $0.247 * * *$ & $(0.043)$ \\
\hline College and above & $0.376 * * *$ & $(0.068)$ & $0.305 * * *$ & $(0.073)$ \\
\hline Logarithm of annual personal income & $0.091 * * *$ & $(0.022)$ & $0.086 * * *$ & $(0.012)$ \\
\hline Hukou status (ref: non-local hukou) & 0.032 & $(0.037)$ & $0.107 * *$ & $(0.054)$ \\
\hline Cut1 & $-1.042 * * *$ & $(0.240)$ & $-1.171 * * *$ & $(0.154)$ \\
\hline Cut2 & $-0.392 *$ & $(0.237)$ & $-0.383^{* *}$ & $(0.151)$ \\
\hline Cut3 & $0.899 * * *$ & $(0.237)$ & $0.898 * * *$ & $(0.151)$ \\
\hline Cut4 & $2.143 * * *$ & $(0.238)$ & $2.164 * * *$ & $(0.152)$ \\
\hline City-level variance & $0.063 * * *$ & $(0.020)$ & $0.049 * * *$ & $(0.016)$ \\
\hline Neighborhood-level variance & $0.045 * * *$ & $(0.014)$ & $0.073 * * *$ & $(0.013)$ \\
\hline Number of individuals & 4731 & & 8530 & \\
\hline Log likelihood & -5679.488 & & $-10,483.653$ & \\
\hline Chi squared & $166.895 * * *$ & & $165.768 * * *$ & \\
\hline
\end{tabular}

$* p<0.10 ; * * p<0.05 ; * * * p<0.01$

\section{Discussion}

We found that commuting time and commuting mode both affected the well-being of urban residents in China. Existing studies mainly focus on the influence of individual socialeconomic characteristics on SWB (Mcbride 2001; Steele and Lynch 2013), and the impact of urban life, especially commuting, on SWB has received insufficient attention in developing countries. In the past 30 years, with the rapid development of the Chinese economy, urban traffic congestion is getting more and more serious and people's commuting times are getting longer and longer. In addition, most jobs in Chinese cities are located in the city centre; but with increasing housing prices in recent years, many wage earners cannot afford homes close to their workplaces and choose to live in suburbs and rural areas that have lower housing prices but are far away from workplaces. The SWB of residents living in urban areas is significantly higher than that of residents living in rural areas. Moreover, longer commute times not only make residents feel physically exhausted but also reduce their SWB. We also found that residents who walk or ride a bike to work 
Table 5 Multilevel mixed-effects ordered probit regression results of commuting impact on residents' subjective well-being based on city urbanization level

\begin{tabular}{|c|c|c|c|c|c|c|}
\hline & \multicolumn{2}{|c|}{$\begin{array}{l}\text { Model 3: } \\
\text { urbanization rate } \\
<50 \%\end{array}$} & \multicolumn{2}{|c|}{$\begin{array}{l}\text { Model 4: } 50 \% \\
<\text { urbanization rate } \\
<70 \%\end{array}$} & \multicolumn{2}{|c|}{$\begin{array}{l}\text { Model 5: } \\
\text { urbanization rate } \\
>70 \%\end{array}$} \\
\hline & Coefficient & SE & Coefficient & $\mathrm{SE}$ & Coefficient & $\mathrm{SE}$ \\
\hline \multicolumn{7}{|l|}{ Traffic variables } \\
\hline Daily commuting time & $-0.118 * * *$ & $(0.033)$ & $-0.079 *$ & $(0.045)$ & -0.019 & $(0.040)$ \\
\hline $\begin{array}{l}\text { Public transport means (ref: } \\
\text { Other transport means) }\end{array}$ & -0.020 & $(0.068)$ & 0.006 & $(0.069)$ & -0.098 & $(0.066)$ \\
\hline $\begin{array}{l}\text { Walk/bicycle (ref: Other } \\
\text { transport means) }\end{array}$ & -0.055 & $(0.037)$ & $-0.093 * *$ & $(0.042)$ & -0.032 & $(0.056)$ \\
\hline \multicolumn{7}{|l|}{ Control variables } \\
\hline Age & 0.002 & $(0.001)$ & $0.004 * *$ & $(0.002)$ & -0.003 & $(0.002)$ \\
\hline Gender (ref: female) & $-0.058^{*}$ & $(0.031)$ & $-0.067 *$ & $(0.036)$ & $-0.142 * * *$ & $(0.043)$ \\
\hline $\begin{array}{l}\text { Marital status (ref: single, } \\
\text { divorced or widowed) }\end{array}$ & $0.196^{* * *}$ & $(0.049)$ & $0.187 * * *$ & $(0.056)$ & $0.444 * * *$ & $(0.060)$ \\
\hline \multicolumn{7}{|c|}{ Educational attainment (ref: primary school and below) } \\
\hline Junior high school & 0.058 & $(0.037)$ & $0.114 * * *$ & $(0.043)$ & $0.138 * *$ & $(0.067)$ \\
\hline Senior high school & $0.193 * * *$ & $(0.050)$ & $0.286^{* * *}$ & $(0.058)$ & $0.188 * *$ & $(0.075)$ \\
\hline College and above & $0.345^{* * *}$ & $(0.065)$ & $0.452 * * *$ & $(0.070)$ & $0.339 * * *$ & $(0.084)$ \\
\hline $\begin{array}{l}\text { Logarithm of annual personal } \\
\text { income }\end{array}$ & $0.082 * * *$ & $(0.015)$ & $0.095 * * *$ & $(0.019)$ & $0.104 * * *$ & $(0.026)$ \\
\hline $\begin{array}{l}\text { Hukou status (ref: non-local } \\
\text { hukou) }\end{array}$ & 0.070 & $(0.049)$ & 0.017 & $(0.056)$ & 0.030 & $(0.050)$ \\
\hline Cut1 & $-1.183 * * *$ & $(0.181)$ & $-1.105 * * *$ & $(0.220)$ & $-0.988 * * *$ & $(0.296)$ \\
\hline Cut2 & $-0.388 * *$ & $(0.178)$ & $-0.415^{*}$ & $(0.216)$ & -0.278 & $(0.293)$ \\
\hline Cut3 & $0.842 * * *$ & $(0.178)$ & $0.933 * * *$ & $(0.216)$ & $1.050 * * *$ & $(0.293)$ \\
\hline Cut4 & $2.074 * * *$ & $(0.179)$ & $2.246^{* * *}$ & $(0.217)$ & $2.275^{* * *}$ & $(0.294)$ \\
\hline City-level variance & $0.057 * * *$ & $(0.018)$ & $0.035^{*}$ & $(0.018)$ & $0.054 * *$ & $(0.024)$ \\
\hline Neighborhood-level variance & $0.059 * * *$ & $(0.013)$ & $0.091 * * *$ & $(0.019)$ & $0.040 * * *$ & $(0.015)$ \\
\hline Number of individuals & 5998 & & 4355 & & 2908 & \\
\hline Log likelihood & -7478.809 & & -5159.533 & & -3510.556 & \\
\hline Chi squared & $117.435 * * *$ & & $120.874 * * *$ & & $126.124 * * *$ & \\
\hline
\end{tabular}

$* p<0.10 ; * * p<0.05 ; * * * p<0.01$

have significantly less sense of happiness than commuters using motor vehicles, which may be related to the imperfect walkways and bicycle lanes in China, where many cities give priority to motor vehicles in road construction (Thomas and Deakin 2008; Pucher et al. 2007). Motorway and pedestrian/bike lanes are often mixed, which reduces the safety and pleasure of people walking or cycling. In addition, traffic pollution has become a major source of urban pollution in China (Fu et al. 2001); therefore, frequent walking or cycling commuting may damage to the health of pedestrians or cyclists. Any of these issues may reduce SWB in urban life. 
Table 6 Multilevel mixed-effects ordered probit regression results of traffic's impact on residents' subjective well-being by hukou status

\begin{tabular}{|c|c|c|c|c|}
\hline & \multicolumn{2}{|c|}{ Model 6: migrants } & \multicolumn{2}{|c|}{ Model 7: local people } \\
\hline & Coefficient & SE & Coefficient & SE \\
\hline \multicolumn{5}{|l|}{ Traffic variables } \\
\hline Daily commuting time & $-0.079 *$ & $(0.048)$ & $-0.081 * * *$ & $(0.025)$ \\
\hline Public transport means (ref: Other transport means) & -0.020 & $(0.072)$ & -0.043 & $(0.046)$ \\
\hline Walk/bicycle (ref: Other transport means) & -0.071 & $(0.058)$ & $-0.062 * *$ & $(0.028)$ \\
\hline \multicolumn{5}{|l|}{ Control variables } \\
\hline Age & -0.002 & $(0.002)$ & $0.003 * *$ & $(0.001)$ \\
\hline Gender (ref: female) & $-0.180^{* * *}$ & $(0.048)$ & $-0.057 * *$ & $(0.023)$ \\
\hline Marital status (ref: single, divorced or widowed) & $0.446 * * *$ & $(0.070)$ & $0.210 * * *$ & $(0.035)$ \\
\hline \multicolumn{5}{|l|}{ Educational attainment (ref: primary school and below) } \\
\hline Junior high school & $0.125 *$ & $(0.075)$ & $0.087 * * *$ & $(0.028)$ \\
\hline Senior high school & $0.157 *$ & $(0.081)$ & $0.243 * * *$ & $(0.037)$ \\
\hline College and above & $0.325 * * *$ & $(0.087)$ & $0.407 * * *$ & $(0.047)$ \\
\hline Logarithm of annual personal income & $0.084 * * *$ & $(0.029)$ & $0.092 * * *$ & $(0.011)$ \\
\hline Cut1 & $-1.101 * * *$ & $(0.320)$ & $-1.166^{* * *}$ & $(0.132)$ \\
\hline Cut2 & -0.413 & $(0.316)$ & $-0.400 * * *$ & $(0.129)$ \\
\hline Cut3 & $0.835 * * *$ & $(0.316)$ & $0.895 * * *$ & $(0.129)$ \\
\hline Cut4 & $1.960 * * *$ & $(0.317)$ & $2.185 * * *$ & $(0.130)$ \\
\hline City-level variance & $0.063 * *$ & $(0.025)$ & $0.056 * * *$ & $(0.013)$ \\
\hline Neighborhood-level variance & $0.059 * * *$ & $(0.023)$ & $0.064 * * *$ & $(0.010)$ \\
\hline Number of individuals & 2397 & & 10,864 & \\
\hline Log likelihood & -3012.558 & & $-13,158.518$ & \\
\hline Chi squared & $92.136 * * *$ & & $265.302 * * *$ & \\
\hline
\end{tabular}

$* p<0.10 ; * * p<0.05 ; * * * p<0.01$

Commute time not only directly affects the SWB of residents but also has an indirect impact on their SWB through job satisfaction, family life satisfaction, residence, and other socioeconomic factors. For example, many people have to travel long distances because of the high housing prices in locations close to workplaces (Logan et al. 2009; Wang and Chai 2009). In this study, we used job satisfaction and family life satisfaction as mediating variables to verify this mediating effect, using Sobel-Goodman mediation tests in Stata (results available upon request). Table 4 shows that only for rural respondents does commuting time significant affect SWB. Therefore, we used the samples of rural respondents to perform the mediation test. We used SWB as the dependent variable, commuting time as the independent variable, and job satisfaction and family life satisfaction as the mediator variables. The results show that commuting time influences residents' job satisfaction and family life satisfaction, which in turn influence their SWB. The mediating effect of job satisfaction is statistically significant, with approximately $54.6 \%$ of the total effect (of commuting time on SWB) being mediated; the mediating effect of family life satisfaction is statistically significant, with approximately $53.4 \%$ of the total 


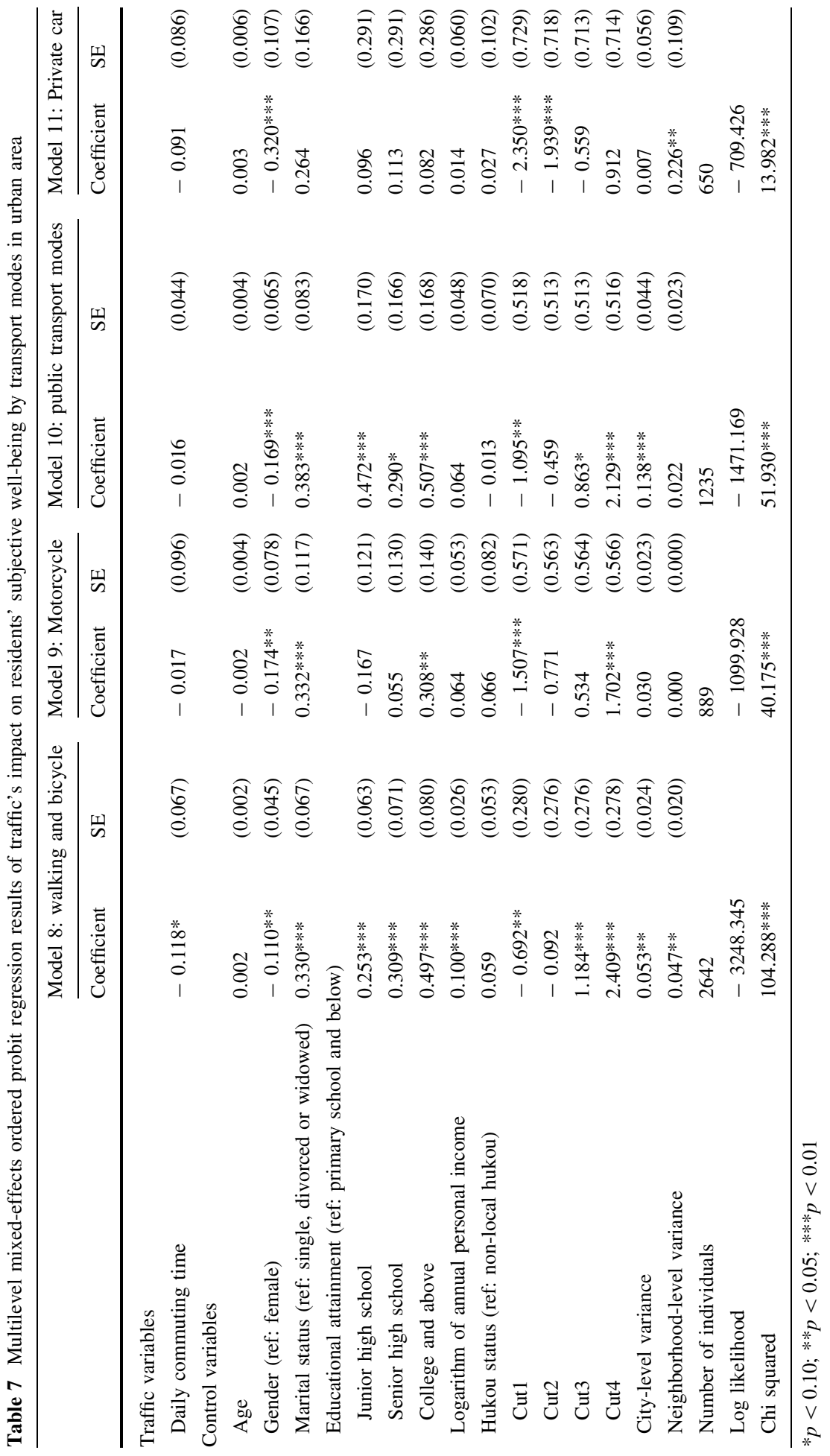




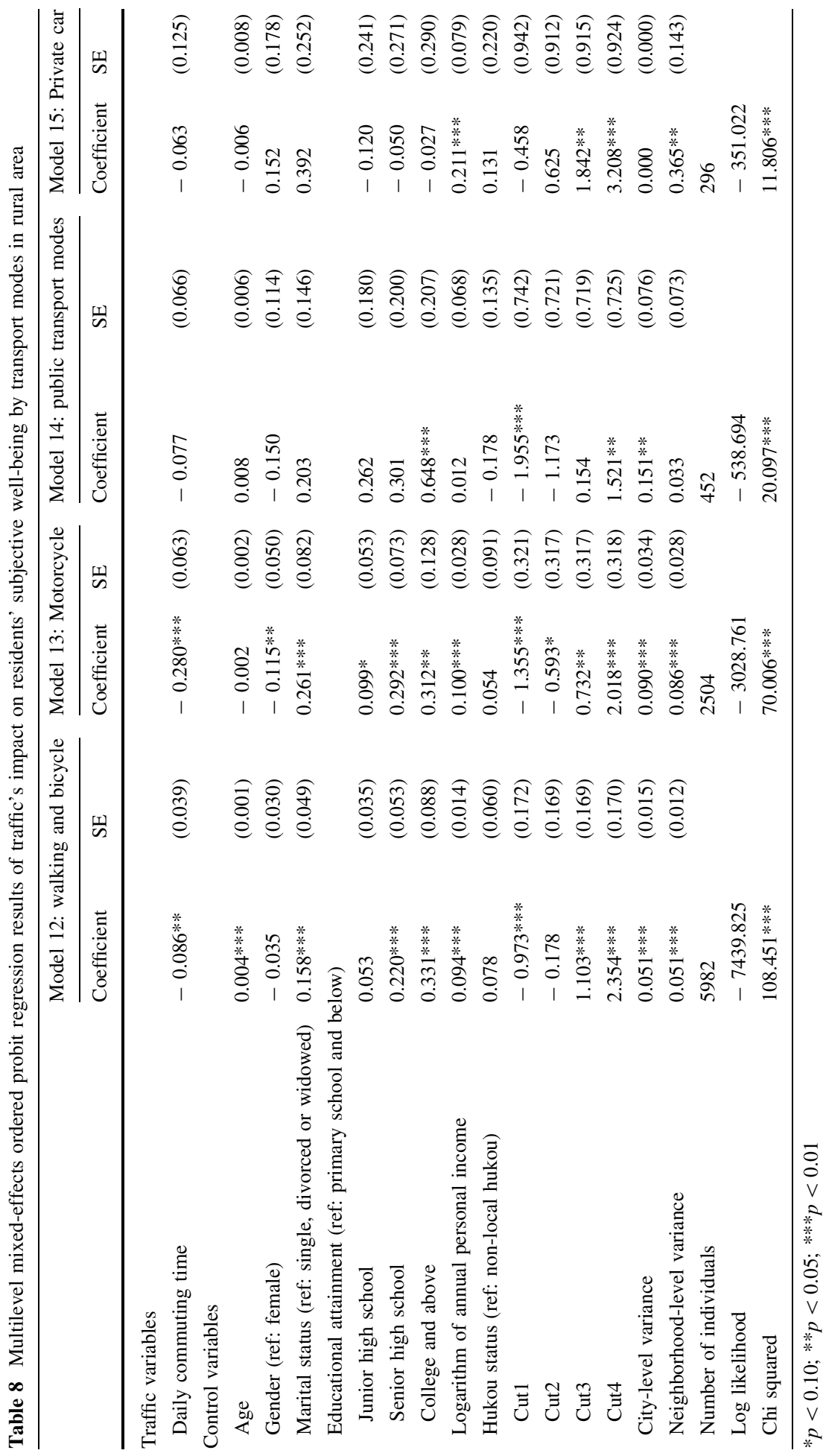


effect of commuting time on SWB. This result aligns with existing studies using lifeoriented approaches (Zhang and Acker 2017).

Looking at differences by urbanization rate, we found that commute time significantly affects SWB in cities with urbanization rates less than $70 \%$. This is related first and foremost to fact that the more urbanized cities in China tend to be those with higher public revenues, which allows for the establishment of multiple transport channels, more frequent and improved public transit service, and better general transportation infrastructure (making commutes more pleasant and traffic less congested). These cities may also feature more jobs and amenities, which might help residents feel more sanguine about enduring their commutes. This latter point is also an important reason why the traffic factors in Table 6 have a less significant impact on the well-being of migrants than of local peoplemigrants generally move from economically backward (often rural) areas to more economically developed (often urban) areas to seek prosperity, and they may be more affected by these urban socioeconomic factors than by traffic factors. Tables 7 and 8 show that the SWB of commuters who walk/ride bikes or ride motorcycles is more affected by commuting time and the transportation environment. During China's rapid development, local governments pursued economic growth based on urban expansion (Wu 2015; Xiao et al. 2006), while ignoring liveability and accessibility. The transportation rights of walkers and cyclists are always neglected in transportation construction. Future traffic construction should give more priority to these vulnerable groups.

To improve commuting efficiency and well-being among residents in Chinese cities, we put forward the following policy suggestions. First, it is necessary to improve job-housing balance and shorten the distance between employment and residence locations. Second, improving the pedestrian/bike lane system and avoiding mixing bike and car lanes will improve satisfaction with walking and bicycling. Third, it is urgent to reduce traffic pollution in order to increase the willingness of residents to commute by bicycle. Before we conclude, several limitations of this study should be registered. First, scholars from different fields (psychology, economics, etc.) have constructed many different SWB scales (Schuur and Kruijtbosch 1995). For instance, Diener (1994) proposed that SWB comprises longer-term levels of pleasant affect, a lack of unpleasant affect, and life satisfaction. However, in this study, due to data limitations, we just used the five-point Likert-type scale to rate respondents' SWB. The CLDS 2014 data provide a rare instance of large-scale national data. It is more accurate to show the relationship between commuting and SWB using data with a large-scale sample size. Therefore, those data were worth using to conduct this research. In future research, SWB can be redefined and reconstructed based on the needs of transportation studies; such a new SWB measure could be more relevant and effective for the field of transportation. Second, as explained in the literature review, SWB is a comprehensive assessment of the current lives of residents. Their SWB could be affected by other socioeconomic factors besides traffic factors, such as housing prices, work pressures, public service availability and quality, and so on. Further, although this study shows that traffic factors (especially commuting time) have a significant impact on SWB, we do not demonstrate that the impact of traffic factors on SWB is stronger than that of other socioeconomic factors, such as health, income, environmental pollution, etc. The impact of traffic on SWB is likely to occur during a short period of time or at a particular time (such as at the time of traffic congestion). Aside from commuting time and transport modes, other traffic factors might have an impact on SWB, such as having a driver's license, auto ownership, length of stay in the residence, proximity to transit options around the neighbourhood, and so forth (Engels and Liu 2011; Bergstad et al. 2011). Third, this study used cross-sectional data, meaning that the relationship between commuting time and 
well-being could not be compared between different years within a given same city. Fourth, although traffic factors did not have a significant impact on residents' well-being in cities with a high urbanization rate, we cannot ignore traffic problems in large cities. For instance, in Beijing, due to high housing price, most working-class people are living far away from their work, and even in other, neighbouring locations (such as Hebei Province, Tianjin, and so on). These people have to spend several hours going to their workplace every day, and irrespective of the results found here that may mitigate perception of its effect on SWB, commuting is still a major urban problem in big cities (with high urbanization rates) in China.

\section{Conclusion}

Commuting negatively affects Chinese people's subjective well-being, especially for residents living in rural areas and walkers/cyclists. Residents commuting by public transport are less subjectively well than those commuting by private car, and those commuting by walking or cycling are less well than others. We also found that residents of cities with high urbanization rates may endure longer commute times than residents of cities with low urbanization rates. In addition, commuting has a greater impact on the SWB of residents with local hukou than on that of migrants.

Open Access This article is distributed under the terms of the Creative Commons Attribution 4.0 International License (http://creativecommons.org/licenses/by/4.0/), which permits unrestricted use, distribution, and reproduction in any medium, provided you give appropriate credit to the original author(s) and the source, provide a link to the Creative Commons license, and indicate if changes were made.

Authors' contributions H. Chen: conceived and designed the study; Z. Zhu and Z. Li: performed the statistical analysis; H. Chen and Z. Zhu: manuscript writing and editing; Y. Liu and J. Zeng: literature search and review, manuscript writing.

\section{References}

Abou-Zeid, M., Ben-Akiva, M.: The effects of social comparisons on commute well-being. Transp. Res. Part A Policy Pract. 45(4), 345-361 (2011)

Abou-Zeid, M., Fujii, S.: Travel satisfaction effects of changes in public transport usage. Transportation 43(2), 301-314 (2016)

Abou-Zeid, M., Witter, R., Bierlaire, M., Kaufmann, V., Ben-Akiva, M.: Happiness and travel mode switching: findings from a Swiss public transportation experiment. Transp. Policy 19(1), 93-104 (2012)

Beige, S., Axhausen, K.W.: The dynamics of commuting over the life course: swiss experiences. Transp. Res. Part A Policy Pract. 104, 179-194 (2017)

Bergstad, C.J., Gamble, A., Gärling, T., et al.: Subjective well-being related to satisfaction with daily travel. Transportation 38(1), 1-15 (2011)

Chan, K.W., Zhang, L.: The hukou system and rural-urban migration in China: processes and changes. China Q. 160, 818-855 (1999)

Chen, H., Jia, B., Lau, S.S.Y.: Sustainable urban form for Chinese compact cities: challenges of a rapid urbanized economy. Habitat Int 32(1), 28-40 (2008)

Choi, J., Coughlin, J., D’Ambrosio, L.: Travel time and subjective well-being. Transp. Res. Rec. J. Transp. Res. Board 2357, 100-108 (2013)

Clark, A.E., Diener, E., Georgellis, Y., Lucas, R.E.: Lags and leads in life satisfaction: a test of the baseline hypothesis. Econ. J. 118(529), F223-F243 (2008a)

Clark, A.E., Frijters, P., Shields, M.A.: Relative income, happiness and utility: an explanation for the Easterlin paradox and other puzzles. J. Econ. Lit. 46(1), 95-144 (2008b) 
Crespo, R.F., Mesurado, B.: Happiness economics, eudaimonia and positive psychology: from happiness economics to floruishing economics. J. Happiness Stud. 16(4), 931-946 (2015)

Creutzig, F., He, D.: Climate change mitigation and co-benefits of feasible transport demand policies in Beijing. Transp. Res. Part D Transp. Environ. 14(2), 120-131 (2009)

Delbosc, A., Nakanishi, H.: A life course perspective on the travel of Australian millennials. Transp. Res. Part A Policy Pract. 104, 319-336 (2017)

Deng, X., Jianmin, X.U., Bo, W.: Traffic countermeasures research for Guangzhou City in traffic mode transferring period after "Motorcycle Forbidden Ban" effect. J. Transp. Syst. Eng. Inf. Technol. 9(4), 145-150 (2009)

Dickerson, A., Hole, A.R., Munford, L.A.: The relationship between well-being and commuting revisited: does the choice of methodology matter? Work. Pap. 49, 321-329 (2014)

Diener, E.: Subjective well-being. Psychol. Bull. 95(3), 542-575 (1984)

Diener, E.: Assessing subjective well-being: progress and opportunities. Soc. Indic. Res. 31(2), 103-157 (1994)

Dolan, P., Peasgood, T., White, M.: Do we really know what makes us happy? A review of the economic literature on the factors associated with subjective well-being. J. Econ. Psychol. 29(1), 94-122 (2008)

Duarte, A., Garcia, C., Giannarakis, G., et al.: New approaches in transportation planning: happiness and transport economics. Netnomics Econ. Res. Electron. Netw. 11(1), 5-32 (2010)

Easterlin, R.A., Angelescu, L.: Happiness and Growth the World Over: Time Series Evidence on the Happiness-Income Paradox. Iza Discussion Papers (4060) (2009)

Engels, B., Liu, G.J.: Social exclusion, location and transport disadvantage amongst non-driving seniors in a Melbourne municipality, Australia. J. Transp. Geogr. 19(4), 984-996 (2011)

Ettema, D., Gärling, T., Eriksson, L., et al.: Satisfaction with travel and subjective well-being: development and test of a measurement tool. Transp. Res. Part F Traffic Psychol. Behav. 14(3), 167-175 (2011)

Ettema, D., Gärling, T., Olsson, L.E., Friman, M.: Out-of-home activities, daily travel, and subjective wellbeing. Transp. Res. Part A Policy Pract. 44(9), 723-732 (2010)

Farrell, S.J., Aubry, T., Coulombe, D.: Neighborhoods and neighbors: do they contribute to personal wellbeing? J. Commun. Psychol. 32(1), 9-25 (2004)

Ferenchak, N.N., Katirai, M.: Commute mode and mental health in major metropolitan areas. Transp. Lett. Int, J. Transp. Res. 7(2), 92-103 (2015)

Frey, B.S., Stutzer, A.: Happiness and Economics: How the Economy and Institutions Affect Human Wellbeing. Princeton University Press, Princeton (2002a)

Frey, B.S., Stutzer, A.: What can economists learn from happiness research? J. Econ. Lit. 40(2), 402-435 (2002b)

Fu, L., Hao, J., He, D., et al.: Assessment of vehicular pollution in China. J. Air Waste Manag. Assoc. 51(5), 658-668 (2001)

Gatersleben, B., Uzzell, D.: Affective appraisals of the daily commute comparing perceptions of drivers, cyclists, walkers, and users of public transport. Environ. Behav. 39(3), 416-431 (2007)

Gerber, P., Ma, T.Y., Klein, O., Schiebel, J., Carpentier-Postel, S.: Cross-border residential mobility, quality of life and modal shift: a Luxembourg case study. Transp. Res. Part A Policy Pract. 104, 238-254 (2017)

Gunatilaka, R.: Does economic growth raise happiness in China? Oxf. Dev. Stud. 39(1), 1-24 (2011)

Kahneman, D., Deaton, A.: High income improves evaluation of life but not emotional well-being. Proc. Natl. Acad. Sci. U.S.A. 107(38), 16489 (2010)

Kahneman, D., Krueger, A.B.: Developments in the measurements of subjective well-being. J. Econ. Perspect. 20(1), 3-24 (2006)

Kahneman, D., Krueger, A.B., Schkade, D.A., Schwarz, N., Stone, A.A.: A survey method for characterizing daily life experience: the day reconstruction method. Science 306(5702), 1776-1780 (2004)

Kroesen, M.: Accessing mediators in the relationship between commute time and subjective well-being. Transp. Res. Rec. J. Transp. Res. Board 2452, 114-123 (2014)

Layard, R.: Measuring subjective well-being. Science 327(5956), 534-535 (2010)

Li, S.M., Liu, Y.: The jobs-housing relationship and commuting in Guangzhou, China: hukou and dual structure 斿. J. Transp. Geogr. 54, 286-294 (2016)

Lin, D., Allan, A., Cui, J.: The impact of polycentric urban development on commuting behaviour in urban China: evidence from four sub-centres of Beijing. Habitat Int. 50, 195-205 (2015)

Linn, J., Wang, Z., Xie, L.: Who will be affected by a congestion pricing scheme in Beijing? Transp. Policy 47, 34-40 (2016)

Liu, Y., Zhang, F., Wu, F., Liu, Y., Li, Z.: The subjective wellbeing of migrants in Guangzhou, China: the impacts of the social and physical environment. Cities 60, 333-342 (2017) 
Logan, J.R., Fang, Y., Zhang, Z.: Access to housing in urban China. Int. J. Urban Reg. Res. 33(4), 914-935 (2009)

Ludwig, J., Duncan, G.J., Gennetian, L.A., Katz, L.F., Kessler, R.C., Kling, J.R., Sanbonmatsu, L.: Neighborhood effects on the long-term well-being of low-income adults. Science 337(6101), 1505-1510 (2012)

Mackerron, G.: Happiness economics from 35000 feet. J. Econ. Surv. 26(4), 705-735 (2012)

MacKerron, G., Mourato, S.: Life satisfaction and air quality in London. Ecol. Econ. 68(5), 1441-1453 (2009)

Mcbride, M.: Relative-income effects on subjective well-being in the cross-section. J. Econ. Behav. Organ. 45(3), 251-278 (2001)

Mccabe, S., Johnson, S.: The happiness factor in tourism: subjective well-being and social tourism. Ann. Tour. Res. 41(1), 42-65 (2013)

Mccabe, S., Joldersma, T., Li, C.: Understanding the benefits of social tourism: linking participation to subjective well-being and quality of life. Int. J. Tour. Res. 12(6), 761-773 (2010)

Morris, E.A.: Access and Outcomes: Transportation, Location, and Subjective Well-being. University of California, Los Angeles (2011)

Morrow, S.L.: The psychological Costs of Commuting: Understanding Relationships between Time, Control, Stress, and Well-being. University of Connecticut, Storrs (2010)

Munford, L.A.: Driving My Life Away? Essays Examining the Impact of Commuting on Income and Wellbeing. University of Sheffield, Sheffield (2014)

Na, T., Chai, Y., Zhang, Y., Sun, D.: Understanding job-housing relationship and commuting pattern in Chinese cities: past, present and future. Transp. Res. Part D Transp. Environ. 52, 562-573 (2017)

Newbold, K.B., Scott, D.M., Burke, C.: Immigrant status and commute distance: an exploratory study based on the greater Golden Horseshoe. Transportation 44(1), 181-198 (2017)

Olsson, L.E., Gärling, T., Ettema, D., et al.: Happiness and satisfaction with work commute. Soc. Indic. Res. 111(1), 255-263 (2013)

Pucher, J., Peng, Z., Mittal, N., et al.: Urban transport trends and policies in China and India: impacts of rapid economic growth. Transp. Rev. 27(4), 379-410 (2007)

Roberts, J., Hodgson, R., Dolan, P.: It's driving her mad: gender difference in the effects of commuting on psychological health. J. Health Econ. 30(5), 1064-1076 (2011)

Schuur, H.V., Kruijtbosch, M.: Measuring subjective well-being. Science 327(5965), 534-535 (1995)

Schwanen, T., Mokhtarian, P.L.: What affects commute mode choice: neighborhood physical structure or preferences toward neighborhoods? J. Transp. Geogr. 13(1), 83-99 (2005)

Smith, O.B.: Peak of the Day or the Daily Grind: Commuting and Subjective Well-being. Portland State University, Portland (2013)

Steele, L.G., Lynch, S.M.: The pursuit of happiness in China: individualism, collectivism, and subjective well-being during China's economic and social transformation. Soc. Indic. Res. 114(2), 441-451 (2013)

Stevenson, B., Wolfers, J.: Economic growth and subjective well-being: reassessing the Easterlin paradox. In: National Bureau of Economic Research, working paper, no. 14282, August (2008)

Stutzer, A., Frey, B.S.: Recent advances in the economics of individual subjective well-being. Soc. Res. 77(2), 679-714 (2010)

Stutzer, A., Frey, B.S.: Stress that doesn't Pay: the commuting paradox. Scand. J. Econ. 110(2), 339-366 (2008)

Sun, B., He, Z., Zhang, T., et al.: Urban spatial structure and commute duration: an empirical study of China. Int. J. Sustain. Transp. 10(7), 638-644 (2016)

Sweet, M., Kanaroglou, P.: Gender differences: the role of travel and time use in subjective well-being. Transp. Res. Part F Traffic Psychol. Behav. 40, 23-34 (2016)

Tella, R.D., Macculloch, R.: Some uses of happiness data in economics. J. Econ. Perspect. 20(1), 25-46 (2006)

Tella, R.D., Macculloch, R.: Gross national happiness as an answer to the Easterlin paradox? Macroeconomics 86(1), 22-42 (2005)

Thomas, A., Deakin, E.: Land use challenges to implementing transit-oriented development in China: case study of Jinan, Shandong Province. Transp. Res. Rec. J. Transp. Res. Board 2077, 80-86 (2008)

Veenhoven, R.: Informed Pursuit of Happiness: what we should know, do know and can get to know. J. Happiness Stud. 16(4), 1035-1071 (2015)

Wang, B., Shao, C., Ji, X.: Dynamic analysis of holiday travel behaviour with integrated multimodal travel information usage: a life-oriented approach. Transp. Res. Part A Policy Pract. 104, 255-280 (2017)

Wang, D., Chai, Y.: The jobs-housing relationship and commuting in Beijing, China: the legacy of Danwei. J. Transp. Geogr. 17(1), 30-38 (2009) 
Wang, L., Xu, J., Qin, P.: Will a driving restriction policy reduce car trips? The case study of Beijing, China. Transp. Res. Part A Policy Pract. 67, 279-290 (2014)

Welsch, H.: Environment and happiness: valuation of air pollution using life satisfaction data. Ecol. Econ. 58(4), 801-813 (2006)

Wheatley, D.: Travel-to-work and subjective well-being: a study of UK dual career household. J. Transp. Geogr. 39, 187-196 (2014)

Wong, D.F.K., Li, C.Y., Song, H.X.: Rural migrant workers in urban China: living a marginalised life. Int. J. Soc. Welf. 16(1), 32-40 (2007)

Wu, F.: Planning for Growth: Urban and Regional Planning in China. Routledge, London (2015)

Xiao, J., Shen, Y., Ge, J., Tateishi, R., Tang, C., Liang, Y., Huang, Z.: Evaluating urban expansion and land use change in Shijiazhuang, China, by using GIS and remote sensing. Landsc. Urban Plan. 75(1-2), 69-80 (2006)

Yang, J., Gakenheimer, R.: Assessing the transportation consequences of land use transformation in urban China. Habitat Int. 31(3-4), 345-353 (2007)

Ye, R., Titheridge, H.: Impact of individuals' commuting trips on subjective well-being evidence from Xi'an, China. J. Transp. Health 2(2), S59 (2015)

Yu, B., Zhang, J., Li, X.: Dynamic life course analysis on residential location choice. Transp. Res. Part A Policy Pract. 104, 281-292 (2017)

Zhang, J., Acker, V.V.: Life-oriented travel behavior research: An overview. Transp. Res. Part A Policy Pract. 104, 167-178 (2017)

Zhang, J.: Life-Oriented Behavioral Research for Urban Policy. Springer, Berlin (2017)

Zhao, J., Lee, J.: Experience or Memory: Happiness of Commuting. Transportation Research Board 92nd Annual Meeting, Washington DC (2013)

Zhao, P., Howden-Chapman, P.: Social inequalities in mobility: the impact of the hukou system on migrants' job accessibility and commuting costs in Beijing. Int. Dev. Plan. Rev. 32(3), 363-384 (2010)

Zhao, P., Lü, B., Roo, G.D.: Impact of the jobs-housing balance on urban commuting in Beijing in the transformation era. J. Transp. Geogr. 19(1), 59-69 (2011a)

Zhao, P., Lu, B., Roo, G.D.: The impact of urban growth on commuting patterns in a restructuring city: evidence from Beijing. Papers Reg. Sci. 90(4), 735-754 (2011b)

Zhao, P., Lü, B., Roo, G.D.: Urban expansion and transportation: the impact of urban form on commuting patterns on the city fringe of Beijing. Environ. Plan. A 42(42), 2467-2486 (2010)

Zhenjun Zhu is a PhD. student in the School of Transportation at Southeast University. His current research interests include transportation modeling and travel demand modeling.

Zhigang Li is a professor in the School of Urban Design at Wuhan University. His research focuses on China's urban development.

Hongsheng Chen is a postdoctor in the School of Geography and Planning at Sun Yat-Sen University. His research focuses on the impact of China's urbanization.

Ye Liu is a professor in the School of Geography and Planning at Sun Yat-Sen University. His research focuses on mobility and urbanization in China.

Jun Zeng is a PhD. student in the School of Transportation at Southeast University. Her current research interests include transportation modeling and simulation. 